\title{
eDNA is a useful tool to evaluate the success of the eradication program of Xenopus laevis in Portugal
}

\author{
Sara Bento $\ddagger$, Bastian Egeter§, Rui Rebelo¥, Cátia Chaves', Joana Pintol \\ ‡ Departamento de Biologia Animal/ Centre for Ecology, Evolution and Environmental Changes Faculdade de Ciências da \\ Universidade de Lisboa, Lisbon, Portugal \\ $\S$ NatureMetrics, London, United Kingdom \\ | CIBIO, Vairão, Portugal
}

Corresponding author: Sara Bento (sarabento94@hotmail.com)

Received: 25 Feb 2021| Published: 04 Mar 2021

Citation: Bento S, Egeter B, Rebelo R, Chaves C, Pinto J (2021) eDNA is a useful tool to evaluate the success of the eradication program of Xenopus laevis in Portugal. ARPHA Conference Abstracts 4: e65055. https://doi.org/10.3897/aca.4.e65055

\begin{abstract}
Biological invasions are widely recognized as a major driver of global biodiversity loss. The most cost-effective answer is often population eradication, while the number of individuals is still limited. The detection of the invasive species at low densities is essential for eradication success, which can be difficult using traditional methods. Environmental DNA (eDNA) can facilitate the detection and monitoring of invasive aquatic species at low densities and can be more sensitive than traditional sampling. The African clawed frog ( Xenopus laevis) is a highly invasive species and has been recorded in several European countries. In Portugal, the species was discovered in 2006 and since 2010, streams of the Lisbon area, suspected or at risk of invasion, are being monitored every year and removal is ongoing. The main method of detection is electrofishing but methods like draining artificial ponds have also been used. This program so far succeeded in containing the spread and reduced frog abundance, although total eradication has not yet been accomplished. To evaluate the success of this program, we collected water samples from fifteen sites. In each site we sampled both pools and rifles to test if lotic microhabitat influences $X$. laevis eDNA detection and concentration. Surface water velocity in each sampling point was also measured. We filtered the samples, extracted DNA from filters,
\end{abstract}


and assayed the extracted DNA for $X$. laevis DNA using quantitative polymerase chain reaction (qPCR). From the fifteen sites sampled, five were positive for $X$. laevis eDNA: four lotic sites and one lentic site. Local eradication success was evident mainly in lentic habitats, and three potential failures were also identified. Finding $X$. laevis at one of these sites only after $X$. laevis eDNA detection was an important contribution of this technique for the success of the control and eradication program. No significative differences were found between lotic microhabitat (riffles and pools) regarding $X$. laevis eDNA concentration and detection but velocity affected the concentration of $X$. laevis eDNA captured. Our results corroborate other studies that recommend eDNA as a complementary sampling approach to other traditional methods and suggests its suitability as a tool for the detection of invasive amphibians.

To be presented as a flash oral presentation at the DNAQUA International Conference First international conference on the use of DNA for water biomonitoring.

\section{Keywords}

Xenopus laevis, invasive species, eradication, species detection, environmental DNA

\section{Presenting author}

Sara Bento

\section{Presented at}

1st DNAQUA International Conference (March 9-11, 2021) 